\title{
Use of the Inferior Whorl for Detecting Age-Related Changes in Human Corneal Subbasal Nerve Plexus with Laser Scanning Confocal Microscopy
}

\author{
Kuiqing Zhao ${ }^{a} \quad$ Hua Yu $^{b} \quad$ Xiaofen Zheng $^{\mathrm{b}} \quad$ Jizhong Yang $^{\mathrm{b}} \quad$ Xiaowu Wang $^{\mathrm{b}}$ \\ Yuping Han ${ }^{b}$ Lijun Jiab Juwei Zhaob \\ aDepartment of Ophthalmology, Yan'an University Affiliated Hospital, Yan'an, China; bShanxi Eye Hospital, Taiyuan, China
}

\section{Keywords}

Inferior whorl · Subbasal nerve plexus · Aging · Laser

scanning confocal microscopy

\begin{abstract}
Purpose: The purpose of this study was to determine the effect of aging on the corneal subbasal nerve plexus (SNP) by employing a wide-field mapping technique of composite images, scanned at the location of a distinctive spiraled subbasal nerve pattern located 1-2 mm inferior to the corneal apex (the inferior whorl) for SNP structural quantification. Materials and Methods: Central corneal tactile sensitivity (CCTS) and inferior whorl length (IWL) were compared among individuals in 3 age-groups (20-39 years, 40-59 years, and 60-79 years). Statistical analyses constituted the Kruskal-Wallis test, one-way analysis of variance (with the post hoc least significant difference test), Spearman correlation coefficient, and linear regression analysis. Results: CCTS remained stable until the age of 50 years, when it began to decrease; the mean CCTS was $58.15 \pm 2.46 \mathrm{~mm}$ in the group aged $20-39$ years, $55.74 \pm 3.85 \mathrm{~mm}$ in the group aged $40-59$ years, and $50.23 \pm 3.27 \mathrm{~mm}$ in the group aged $60-79$ years. IWL decreased with increasing age, with a corresponding lin-
\end{abstract}

karger@karger.com

(c) 2020 S. Karger AG, Basel

www.karger.com/ore

Karger" ear decline of $0.2088 \mathrm{~mm} / \mathrm{mm}^{2}$ per year, and the mean IWL was $25.43 \pm 4.50 \mathrm{~mm} / \mathrm{mm}^{2}$ in the group aged $20-39$ years, $22.71 \pm 6.19 \mathrm{~mm} / \mathrm{mm}^{2}$ in the group aged $40-59$ years, and $18.60 \pm 4.21 \mathrm{~mm} / \mathrm{mm}^{2}$ in the group aged $60-79$ years. Conclusion: Our work provided a more accurate and repeatable method for corneal nerve analysis using laser scanning confocal microscopy. By using this technique, we confirmed that aging is associated with progressive reduction in subbasal nerve length.

(c) 2020 S. Karger AG, Basel

\section{Introduction}

In vivo confocal microscopy (IVCM) is a rapid, noninvasive, and reiterative technique that enables high-resolution microstructural evaluation of the human cornea. In order to improve image quality, IVCM has undergone extensive development since the principle was first described in 1986. Recently, a coherent light source has been developed to generate the laser scanning confocal microscopy

Kuiqing Zhao and Hua Yu contributed equally to this work.
Correspondence to:

Xiaofen Zheng, zxffd2000@126.com 
(LSCM). With the aid of LSCM, our knowledge of corneal nerves in health and disease, especially with regard to the corneal subbasal nerve plexus (SNP), has been largely enhanced. The SNP is confined to a thin plexus located at the junction of the basal epithelium and the anterior aspect of Bowman's layer. This plexus is regarded as vital to the health and functioning of the epithelium, tear film, and cornea because it serves as a source of epithelial innervation with intraepithelial nerve endings terminating at the ocular surface $[1,2]$. Alterations of the SNP affect physiological aspects of epithelial cell metabolism and proliferation, including permeability, migration, and mitosis; these changes increase the risks of recurrent erosion, impaired wound healing, and infection [3]. Aging is an important factor that renders the cornea susceptible to harmful environmental influences and diseases [4]. Although corneal sensitivity has been shown to decline with age $[5,6]$, the specific effect of aging on the SNP remains a controversial topic. In previous studies, images of the SNP around the central cornea were often used for quantitative analysis. However, the specific corneal area captured by LSCM was not definitively determined in those studies, as LSCM can only capture a small area and no markers of the corneal center are available during measurement. Therefore, the areas used for this assessment may vary among patients and may change over time in the same patient; such variations can result in wide discrepancies, particularly in longitudinal and interventional studies.

Patel and McGhee [7] recently demonstrated that the corneal subbasal nerves anastomose extensively and form a dense homogenous nerve network that radiates toward a whorl-like assembly (the inferior whorl), typically 1-2 mm inferior to the corneal apex. Because of its unique pattern and relatively fixed location, the inferior whorl constitutes a distinct corneal landmark for accurate measurement of the SNP [8]. Therefore, this site was selected in the current study to quantify the structural status of the subbasal nerve, with the aim of determining the effect of aging on the SNP.

\section{Material and Methods}

\section{Participants}

This study adhered to the tenets of the Declaration of Helsinki and was approved by the Ethics Committee of Shanxi Eye Hospital. Written informed consent was obtained from each participant after detailed explanation of the study. Individuals (2080 years of age) were recruited from November 2018 to February 2019 in Taiyuan, China. The exclusion criteria were as follows: a history of contact lens wear, ocular surgery or trauma, and the presence of ocular disease (such as pterygium, glaucoma, and keratoconus) or systemic disease that might affect the cornea or nervous system (such as diabetes, alcoholism, medications, cancer, kidney disease, liver disease, connective tissue disorders, rheumatoid arthritis, vitamin $B_{12}$ deficiency, or idiopathic small fiber neuropathy). The modified neuropathy disability score and ocular surface disease index questionnaire were used to ensure that participants had no signs or symptoms of neuropathy and dry eye. After the application of these screening criteria, 76 healthy individuals were included in the study and divided into 3 age-groups: $20-39$ years (group A), 40-59 years (group B), and 60-79 years (group C).

\section{Corneal Sensitivity Measurement}

Central corneal tactile sensitivity (CCTS) was evaluated with the Cochet-Bonnet esthesiometer (Luneau Ophtalmologie, Chartres, France), which consists of a nylon monofilament with a 0.12 $\mathrm{mm}$ diameter that can be modified in length from 5 to $60 \mathrm{~mm}$ to achieve different degrees of pressure application to the cornea. All participants were examined in a room with a controlled atmosphere (humidity of $40-60 \%$ and temperature of $25-30^{\circ} \mathrm{C}$ ) by the same experienced observer between 10:00 a.m. and 12:00 p.m. to avoid diurnal variation. The participants were seated and instructed to visually fixate on a target straight ahead. The nylon filament was held perpendicular to the stimulated corneal point, and the eye blink reflex was regarded as an indicator of the perception of corneal sensation. The test began with the filament extended to $60 \mathrm{~mm}$ at the lowest pressure; if no response was elicited, the filament was shortened by $5 \mathrm{~mm}$ increments and the test was repeated until a response was observed. Five measurements were taken for each participant when at least 3 measurements resulted in a blink reflex, the length of the filament was recorded, and the mean of the measurements was used in statistical analysis.

\section{Laser Scanning Confocal Microscopy}

Laser scanning was performed on the right eye of each participant using the Heidelberg Retina Tomograph II, Rostock Corneal Module (Heidelberg Engineering GmbH, Heidelberg, Germany). This examination provides two-dimensional images that consist of $384 \times 384$ pixels, with a view field of $400 \times 400 \mu \mathrm{m}$. A drop of Vidisic (carbomer eye drops, $0.2 \%$; Bausch \& Lomb/Dr. Mann Pharma, Berlin, Germany) was used as a coupling agent between the applanating lens cap and the cornea. Each eye undergoing scanning was anesthetized with $0.2 \%$ anesthetic proparacaine hydrochloride (S.A. Alcon-COUVREUR N.V.), and participants were instructed to fixate the contralateral eye on a fixation lamp during examination. At the beginning of scanning, the fixation lamp was placed slightly above the corneal apex to capture images of the whorl-like region, as it lies in the inferocentral portion of the cornea. If the distinctive whorl-like pattern was identified, the fixation lamp was moved in a grid pattern [7] to permit large-scale visualization of the corneal SNP over an area up to $2 \times 2 \mathrm{~mm}$, using the section mode.

Single images of the whorl-like region were manually arranged into a contiguous montage using Adobe Photoshop CC 2017 (Adobe Systems Incorporated, California, US). A standard frame size of $700 \times 700 \mu \mathrm{m}$ was selected in the center of the whorl-like region. All nerve fibers and branches in the defined frame were traced semiautomatically using Neuron-J (a plug-in module for Image-J; Erik Meijering, Rotterdam, the Netherlands) to determine the inferior whorl length (IWL), which constituted the total nerve length per square millimeter in the whorl-like region. 
Table 1. Characteristics of the study participants

\begin{tabular}{lllll}
\hline Characteristic & $\begin{array}{l}\text { Group A } \\
(20-39 \text { years })\end{array}$ & $\begin{array}{l}\text { Group B } \\
(40-59 \text { years })\end{array}$ & $\begin{array}{l}\text { Group C } \\
(60-79 \text { years })\end{array}$ & $p$ (group difference) \\
\hline Participants, $n$ & 27 & 27 & 22 & - \\
Age, years & $30.52 \pm 4.75$ & $49.00 \pm 4.64$ & $63.45 \pm 3.58$ & $<0.0001^{*}$ \\
Sex, male/female & $10 / 17$ & $11 / 16$ & $9 / 13$ & $0.9492^{\dagger}$ \\
CCTS, mm & $58.15 \pm 2.46$ & $55.74 \pm 3.85$ & $50.23 \pm 3.27$ & $<0.0001^{\#}$ \\
IWL, $\mathrm{mm} / \mathrm{mm}^{2}$ & $25.43 \pm 4.50$ & $22.71 \pm 6.19$ & $18.60 \pm 4.21$ & $<0.0001^{*}$ \\
\hline
\end{tabular}

CCTS, central corneal tactile sensitivity; IWL, inferior whorl length. Data are means \pm standard deviation. * One-way analysis of variance test. ${ }^{\dagger}$ Kruskal-Wallis test. ${ }^{*} \chi^{2}$ test.

\section{Statistical Analysis}

Statistical analyses were performed with IBM SPSS Statistics (version 17.0; IBM Corp., Armonk, NY). Data are represented as mean \pm standard deviation. Corneal sensitivity differences among the 3 age-groups were evaluated using the Kruskal-Wallis test, and the Spearman correlation coefficient was used for correlation analysis. One-way analysis of variance (with the post hoc least significant difference test) was used to compare IWL among groups, and age trends were investigated using linear regression analyses. Differences with $p<0.05$ were considered to be statistically significant.

\section{Results}

The right eyes of 76 participants (age range 25-72 years) were analyzed. The mean ages were $30.52 \pm 4.75$ years in group A (10 males and 17 females), $49.00 \pm 4.64$ years in group B (11 males and 16 females), and $63.45 \pm$ 3.58 years in group $\mathrm{C}$ ( 9 males and 13 females). There were no significant differences in sex composition between groups $\left(\gamma^{2}=0.104, p=0.949\right)$. Participants' characteristics are presented in Table 1.

All data were plotted and age-related changes in CCTS were fitted to a parabolic curve that exhibited an initial plateau until the age of 50 years and sharply decreased thereafter (Fig. 1). There was no significant difference in CCTS between group A and group B ( $p=0.1119)$, but a significant decrease was observed in participants aged $\geq 60$ years when compared with those aged 20-39 years and 40-59 years $(p<0.0001$ and $p=0.0002$, respectively). Spearman correlation coefficient analysis showed a negative correlation between age and CCTS $(r=-0.6751, p<0.0001)$.

Representative LSCM images for each age-group are presented in Figure 2. Parametric analysis of these images demonstrated that IWL showed a decreasing trend associated with increasing age $(p<0.0001)$; linear regression analysis also revealed a significant correlation between age and IWL $\left(R^{2}=0.2614, p<0.0001\right)$ with a reduction of 0.2088

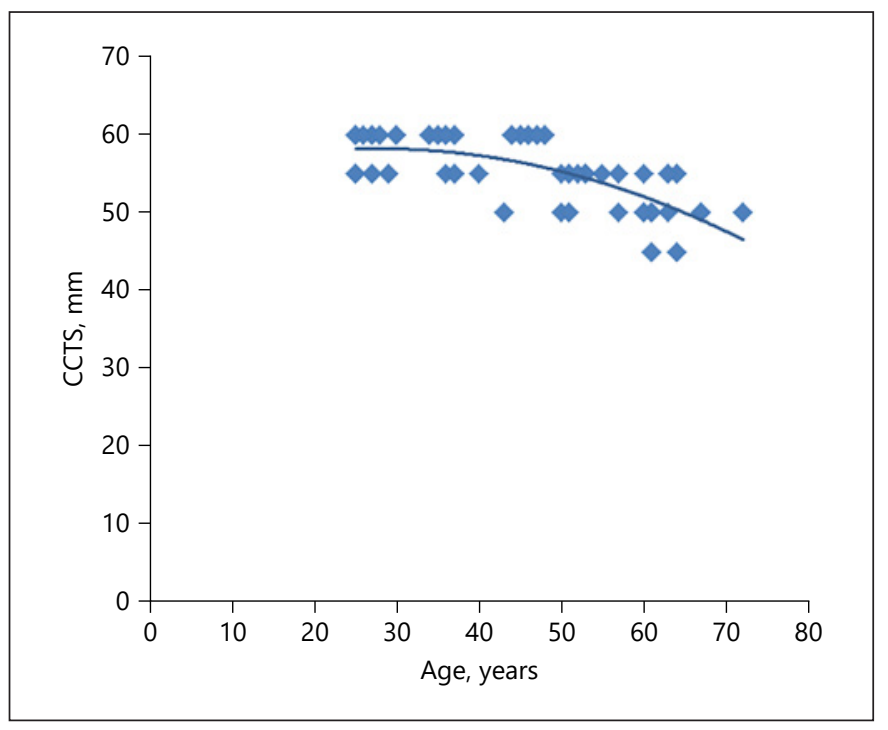

Fig. 1. CCTS changes with age. Parabolic fitted curve shows agerelated variation with stability until the age of 50 years, and followed by reduction with a steep slope. CCTS, central corneal tactile sensitivity.

$\mathrm{mm} / \mathrm{mm}^{2}$ per year (Fig. 3). There was no significant difference in IWL between group A and group B $(p=0.1611)$, but a significant decrease was observed in participants aged $\geq 60$ years when compared with those aged 20-39 years and 4059 years $(p<0.0001$ and $p=0.0188$, respectively).

\section{Discussion}

The process of aging causes structural and functional changes in the cornea and has been associated with delayed wound healing activities and reduced resistance to infections [3]. Corneal sensitivity is an indicator of corneal health status, which permits evaluation of the response to external 

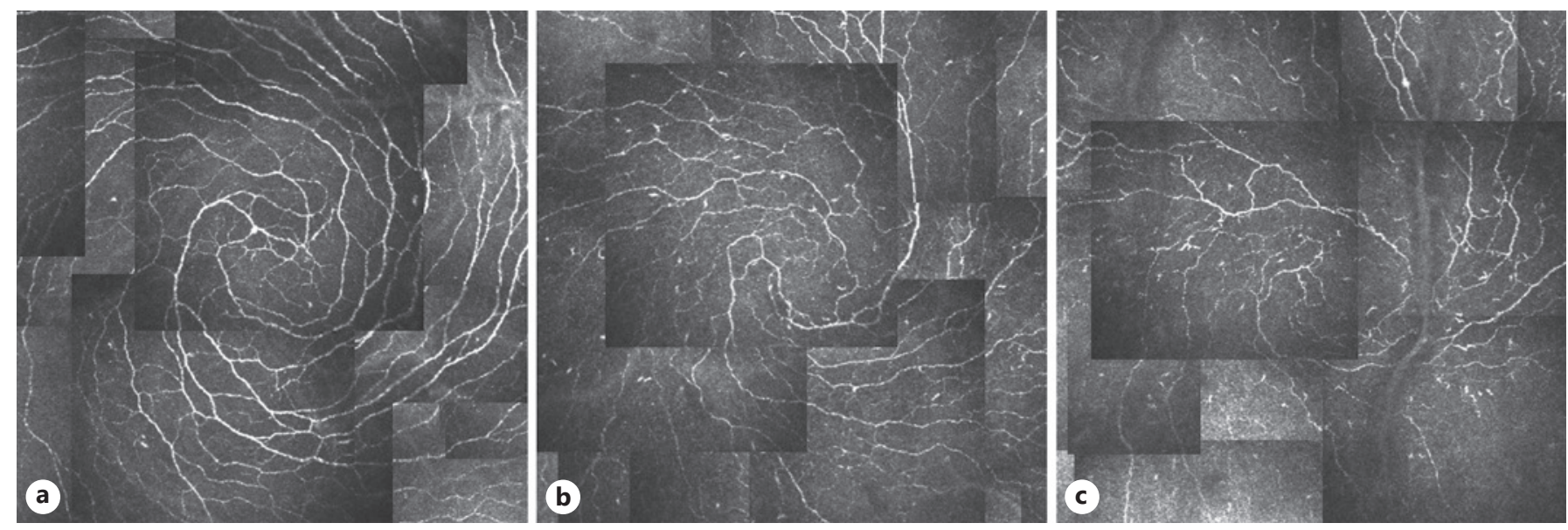

Fig. 2. Corneal SNP morphology of each age-group at the inferior whorl. a 20-39 years (group A). b 40-59 years (group B). c 60-79 years (group C). Visual field was $700 \times 700 \mu \mathrm{m}$. SNP, subbasal nerve plexus.

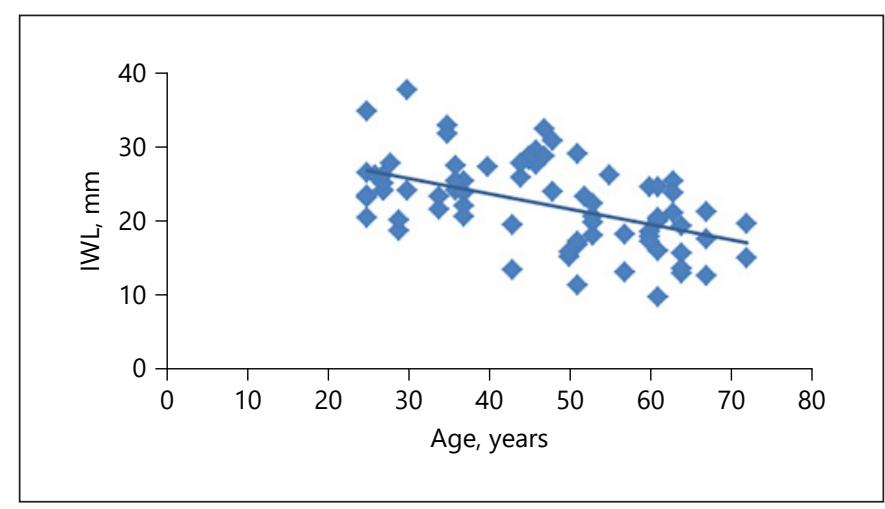

Fig. 3. Regression plots for IWL and age. Linear regression line shows that IWL declines with age. IWL, inferior whorl length.

insults or postsurgical healing. The results of the current study confirmed earlier findings regarding age-related modifications of central corneal sensitivity $[5,6]$, indicated a nonlinear correlation fitted by a parabolic curve, and demonstrated that CCTS remained stable until the age of 50 years when it began to decrease.

Corneal sensitivity provides an important measurement of the corneal nerve function, but the relationships between the corneal nerve structure and function remain complex and variable across different situations. In terms of CCTS, Cochet-Bonnet esthesiometers are thought to only quantify the function of $A \delta$ fibers [9], while the human corneal subbasal nerves contain both myelinated $A \delta$ and unmyelinated $\mathrm{C}$ fibers [3]; thus, this measurement method may yield biased estimations of the status of corneal innervation based on corneal sensation.
The introduction of IVCM has provided a new method for direct evaluation of corneal innervation. By using IVCM, a growing number of studies have shown that SNP may be a suitable proxy for assessing corneal health or pathology $[10,11]$; it may even be used to gauge the severity of non-ocular conditions, such as peripheral neuropathy in diabetes mellitus [12] or rheumatoid arthritis [13]. Given the utility of SNP in screening, detection, and monitoring of corneal and systemic neuropathies, it is important to understand how aging might affect this nerve plexus because the determination of normal values at different ages is crucial for establishing a baseline against which a "pathologic" level can be defined. However, there is inconsistency in the literature with respect to the relationship between age and neural morphometric changes in the SNP. While a number of studies have reported no significant age-related changes in subbasal nerve morphology $[1,14,15]$, others have reported age-related reductions in nerve density [16-18]. Differences in methodologies, including types of instruments (slit scanning or laser scanning confocal system), numbers of participants (in terms of age distribution), and protocols used for image acquisition and analysis (volume or sequence scan mode, dense or representative region, number of images to analyze, number and experience of observers, masking, and randomization), have resulted in wide discrepancies and bias-prone estimates. Some of these problems derive from the poor topographic reproducibility of IVCM, which causes difficulty in ensuring that a consistent location is captured in different patients at a single time point or in the same patient at multiple time points. In 2005, Patel and McGhee [7] first demonstrated that the SNP has 
a whorl-like complex (i.e., the inferior whorl), which is a distinctive spiraled (clockwise or counterclockwise) subbasal nerve fiber pattern located 1-2 $\mathrm{mm}$ inferior to the corneal apex. Because of its unique pattern, the inferior whorl has been proposed as a more reliable landmark for longitudinal and interventional assessment of the SNP [8]. Therefore, the current study focused on this site for subbasal nerve structural quantification, and our results showed that the IWL of the 3 age-groups was $25.43 \pm 4.50$, $22.71 \pm 6.19$, and $18.60 \pm 4.21 \mathrm{~mm} / \mathrm{mm}^{2}$, respectively.

Another limitation that prohibits IVCM from gaining wider clinical application is that it involves a small imaging area: the typical field of view with LSCM is $400 \times 400$ $\mu \mathrm{m}$ (only $0.15 \%$ of the total corneal area), which does not provide the necessary basis for statistically firm conclusions concerning morphometric plexus alterations. To address this limitation, multiple representative images have been proposed for analysis; however, this approach is subjective, nonstandardized, prone to image selection bias, and has poor reproducibility [19,20]. More recently, a wide-field mapping approach that involves composite images of a larger area has been employed for SNP assessment $[7,21-25]$. This technique could reduce the variability inherent in image sampling and enable investigation of global nerve architecture in a manner that cannot be achieved with the single field of view in IVCM images. Using a method described in a previously published study [7], we successfully performed wide-field composite images of the SNP and demonstrated that the subbasal nerve length decreased with increasing age (linear reduction of $0.2088 \mathrm{~mm} / \mathrm{mm}^{2}$ per year). This finding is in accordance with the results of Niederer's study, which showed a reduction of $0.9 \%$ per year [18]; however, it conflicts with the findings of Parissi et al. [16] and Tavakoli et al.'s [26] studies which showed reductions of $0.25-0.30 \%$ per year and $0.060 \mathrm{~mm} / \mathrm{mm}^{2}$ per year, respectively. Differences in scanning sites and image selection methods may explain this discrepancy. Furthermore, our results showed that at the age of 60 years, SNP structural loss becomes significant in comparison with those $<60$ years, which may partly explain the mechanism of delayed wound healing and reduced corneal sensation in elderly individuals.

To the best of our knowledge, this is the first use of wide-field composite images of the inferior whorl to investigate age-related changes in the SNP. Our work provided a more accurate and repeatable method for corneal nerve analysis using LSCM, and the results demonstrated an age-related reduction of subbasal nerve length, constituting a linear decline of $0.2088 \mathrm{~mm} / \mathrm{mm}^{2}$ per year with a threshold age of 60 years.

Age-Related Changes in Human Corneal

Subbasal Nerve Plexus

\section{Statement of Ethics}

This study adhered to the tenets of the Declaration of Helsinki and was approved by the Ethics Committee of Shanxi Eye Hospital (approval number 201804b). Written informed consent was obtained from all patients or their families for the publication of this report and associated images.

\section{Conflict of Interest Statement}

The authors have no conflicts of interest to disclose.

\section{Funding Sources}

This research received no specific grant from any funding agency in the public, commercial, or not-for-profit sectors.

\section{Author Contributions}

Kuiqing Zhao designed the study and contributed to the analysis of data. Hua $\mathrm{Yu}$ contributed to data collection and wrote the initial draft of the manuscript. Xiaofen Zheng contributed to the analysis and interpretation of data, and critically reviewed the manuscript. All other authors contributed to data collection and assisted in the preparation of the manuscript. All authors approved the final version of the manuscript and agree to be accountable for all aspects of the work in ensuring that questions related to the accuracy or integrity of any part of the work are appropriately investigated and resolved.

\section{Data Availability Statement}

The data are not available for public access because of patient privacy concerns but are available from the corresponding author on reasonable request.

\section{References}

1 Marfurt CF, Cox J, Deek S, Dvorscak L. Anatomy of the human corneal innervation. Exp Eye Res. 2010;90:478-92.

2 Guthoff RF, Wienss H, Hahnel C, Wree A. Epithelial innervation of human cornea: a three-dimensional study using confocal laser scanning fluorescence microscopy. Cornea. 2005;24:608-13.

3 Muller LJ, Marfurt CF, Kruse F, Tervo TM Corneal nerves: structure, contents and function. Exp Eye Res. 2003;76:521-42.

4 Gipson IK. Age-related changes and diseases of the ocular surface and cornea. Invest Ophthalmol Vis Sci. 2013;54:ORSF48-53.

5 Roszkowska AM, Colosi P, Ferreri FM, Galasso S. Age-related modifications of corneal sensitivity. Ophthalmologica. 2004;218(5): 350-5. 
6 Mirzajan A, Khezri F, Jafarzadehpur E, Karimian F, Khabazkhoob M. Normal corneal sensitivity and its changes with age in Tehran, Iran. Clin Exp Optom. 2015;98(1):54-7.

7 Patel DV, McGhee CN. Mapping of the normal human corneal sub-basal nerve plexus by in vivo laser scanning confocal microscopy. Invest Ophthalmol Vis Sci. 2005;46:4485-8.

8 Utsunomiya T, Nagaoka T, Hanada K, Omae $\mathrm{T}$, Yokota $\mathrm{H}$, Abiko A, et al. Imaging of the corneal subbasal whorl-like nerve plexus: more accurate depiction of the extent of corneal nerve damage in patients with diabetes. Invest Ophthalmol Vis Sci. 2015;56:5417-23.

9 Belmonte C, Acosta MC, Gallar J. Neural basis of sensation in intact and injured corneas. Exp Eye Res. 2004;78:513-25.

10 Patel DV, McGhee CN. In vivo confocal microscopy of human corneal nerves in health, in ocular and systemic disease, and following corneal surgery: a review. Br J Ophthalmol. 2009;93:853-60.

11 Cruzat A, Pavan-Langston D, Hamrah P. In vivo confocal microscopy of corneal nerves: analysis and clinical correlation. Semin Ophthalmol. 2010;25:171-7.

12 Pritchard N, Edwards K, Shahidi AM, Sampson GP, Russell AW, Malik RA, et al. Corneal markers of diabetic neuropathy. Ocul Surf. 2011;9:17-28.
13 Villani E, Galimberti D, Viola F, Mapelli C, Del Papa N, Ratiglia R. Corneal involvement in rheumatoid arthritis: an in vivo confocal study. Invest Ophthalmol Vis Sci. 2008;49: 560-4.

14 Erie JC, McLaren JW, Hodge DO, Bourne WM. The effect of age on the corneal subbasal nerve plexus. Cornea. 2005;24:705-9.

15 Patel DV, Tavakoli M, Craig JP, Efron N, McGhee CN. Corneal sensitivity and slit scanning in vivo confocal microscopy of the subbasal nerve plexus of the normal central and peripheral human cornea. Cornea. 2009;28: 735-40.

16 Parissi M, Karanis G, Randjelovic S, Germundsson J, Poletti E, Ruggeri A, et al. Standardized baseline human corneal subbasal nerve density for clinical investigations with laser-scanning in vivo confocal microscopy. Invest Ophthalmol Vis Sci. 2013;54:7091102.

17 He J, Bazan NG, Bazan HE. Mapping the entire human corneal nerve architecture. Exp Eye Res. 2010;91:513-23.

18 Niederer RL, Perumal D, Sherwin T, McGhee $\mathrm{CN}$. Age-related differences in the normal human cornea: a laser scanning in vivo confocal microscopy study. Br J Ophthalmol. 2007;91: $1165-9$.

19 Hertz P, Bril V, Orszag A, Ahmed A, Ng E, Nwe P, et al. Reproducibility of in vivo corneal confocal microscopy as a novel screening test for early diabetic sensorimotor polyneuropathy. Diabet Med. 2011;28:1253-60.
20 Winter K, Scheibe P, Köhler B, Allgeier S, Guthoff RF, Stachs O. Local variability of parameters for characterization of the corneal subbasal nerve plexus. Curr Eye Res. 2016; 41(2):186-98.

21 Allgeier S, Zhivov A, Eberle F, Koehler B, Maier $S$, Bretthauer $G$, et al. Image reconstruction of the subbasal nerve plexus with in vivo confocal microscopy. Invest Ophthalmol Vis Sci. 2011;52:5022-8.

22 Zhivov A, Blum M, Guthoff R, Stachs O. Realtime mapping of the subepithelial nerve plexus by in vivo confocal laser scanning microscopy. Br J Ophthalmol. 2010;94:1133-5.

23 Edwards K, Pritchard N, Gosschalk K, Sampson GP, Russell A, Malik RA, et al. Wide-field assessment of the human corneal subbasal nerve plexus in diabetic neuropathy using a novel mapping technique. Cornea. 2012;31:1078-82.

24 Turuwhenua JT, Patel DV, McGhee CN. Fully automated montaging of laser scanning in vivo confocal microscopy images of the human corneal subbasal nerve plexus. Invest Ophthalmol Vis Sci. 2012;53:2235-42.

25 Yokogawa H, Kobayashi A, Sugiyama K. Mapping of normal corneal K-structures by in vivo laser confocal microscopy. Cornea. 2008;27:879-83.

26 Tavakoli M, Ferdousi M, Petropoulos IN, Morris J, Pritchard N, Zhivov A, et al. Normative values for corneal nerve morphology assessed using corneal confocal microscopy: a multinational normative data set. Diabetes Care. 2015;38(5):838-43. 